
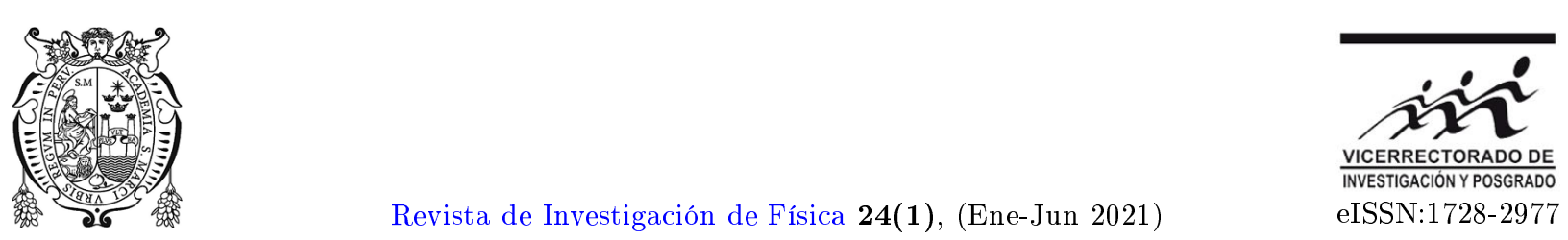

\title{
Desarrollo de un espectrómetro de impedancia eléctrica portátil para análisis y caracterización del tejido sanguíneo
}

\author{
DChristian Lexequías ${ }^{* 1}$, Bryan Urcia ${ }^{1}$, Betty Franco ${ }^{2}$, Oscar Baltuano ${ }^{1}$ y Galo Patiño ${ }^{1}$ \\ ${ }^{1}$ Universidad Nacional Mayor de San Marcos, Grupo de investigación INFISA, Lima, Perú \\ ${ }^{2}$ Grupo de Investigación en Ciencia, Tecnología y Medio Ambiente, I.E. Gabriela Mistral, Lima, Perú
}

Recibido 05 setiembre 2020 - Aceptado 31 octubre 2020

\begin{abstract}
Resumen
Haciendo uso de un Analog Discovery 2 como base para el desarrollo de un equipo de espectroscopia de impedancia eléctrica (EIE) y la adaptación de las tiras reactivas Accu-Chek@ como electrodo de trabajo para realizar lecturas en muestras de tejido sanguíneo humano, fue posible obtener los espectros correspondientes de Nyquist para el tejido mencionado. Estos resultados indican una posible correspondencia en la señal de impedancia eléctrica observada en personas con una cantidad de hemoglobina $(\mathrm{Hb})$ de $14.8 \mathrm{~g} / \mathrm{dL}, 14.7 \mathrm{~g} / \mathrm{dL}, 14,6 \mathrm{~g} / \mathrm{dL}$ y $13,7 \mathrm{~g} / \mathrm{dL}$. También fue posible simular con los datos obtenidos el gráfico probable de una persona con $12.7 \mathrm{~g} / \mathrm{dL}$ de hemoglobina en sangre, que corresponde a un paciente con anemia. El presente articulo analiza la viabilidad del desarrollo de un nuevo método de diagnóstico menos invasivo para la enfermedad de anemia el cual se viene llevando a cabo en el Laboratorio de Biofísica de la Facultad de Ciencias Físicas - UNMSM.
\end{abstract}

Palabras clave: Impedancia eléctrica, hemoglobina, Nyquist, tejido sanguíneo, diagnóstico.

Development of a portable electrical impedance spectrometer for the analysis and characterization of blood tissue

\begin{abstract}
Using an Analog Discovery 2 as the basis for the development of an electrical impedance spectroscopy (EIS) kit and the adaptation of the $A c c u-C h e k \Omega$ test strips as a working electrode to perform readings on samples of human blood tissue, it is possible to obtain the corresponding Nyquist spectra for the mentioned tissue. These results indicate a possible correspondence in the electrical impedance signal observed in people with a hemoglobin amount of $14.8 \mathrm{~g} / \mathrm{dL}, 14.7 \mathrm{~g} / \mathrm{dL}, 14.6 \mathrm{~g} / \mathrm{dL}$ and $13.7 \mathrm{~g} / \mathrm{dL}$. It was also possible to simulate with the data obtained the probable graph of a person with $12.7 \mathrm{~g} / \mathrm{dL}$ of hemoglobin in blood, which corresponds to a patient with anemia. This article analyzes the viability of developing a new less invasive diagnostic method for anemia disease, which is taking place in the Biophysics Laboratory of the Faculty of Physical Sciences - UNMSM.
\end{abstract}

Keywords: Electrical impedance, hemoglobin, Nyquist, blood tissue, diagnosis.

\section{Introducción}

La espectroscopía de impedancia eléctrica (EIE) es una técnica que se ha utilizado ampliamente para la caracterización de electrolitos [Got96] [And05], tamizaje de productos alimenticios [Mas10], estudios de corrosión [Cal80], control de calidad de recubrimientos y superficies [Gom07], caracterización de organismos microbiológicos como E. Coli [Bal19] y Salmonella [Gri04].
Además, se ha empleado para el estudio de tejidos, como la sangre [Ber05a] [Ber05b], el tejido adiposo [Alm12], etc. Debido a estas investigaciones previas, este método resulta factible para el diagnóstico rápido de diferentes enfermedades que afectan el tejido sanguíneo.

Se estima que en el Perú cuatro de cada diez niños menores de tres años tienen anemia, siendo $40.1 \%$ el último índice registrado por el Ministerio de Desarrollo e Inclusión Social-MIDIS para personas que padecen

\footnotetext{
*christian.lexequias@unmsm.edu.pe
} 
de prevalencia de anemia a nivel nacional [Ine19]. Para diagnosticar esta enfermedad los profesionales de la salud utilizan un método que consta de la extracción del tejido sanguíneo, el cual pasa por un proceso que mide la absorbancia de la sangre total en un punto isosbéstico, esto quiere decir la longitud de onda concreta o frecuencia en la que la absorbancia total determina la concentración de hemoglobina ( $\mathrm{Hb} / \mathrm{HbO} 2$ ) [Cau85]. Este resulta ser un método invasivo que puede provocar efectos adversos en los pacientes como la punción accidental de una arteria debido a una mala praxis, formación de hematomas originados por el desbordamiento de la sangre en el tejido antes, durante o después de la punción, o una lesión nerviosa debido a la inserción muy rápida o profunda de la aguja [And10]. Debido a este alto porcentaje de personas anémicas en el país y las deficiencias para el análisis in situ de este tipo de enfermedad en áreas rurales alejadas de la capital es que surge la idea de desarrollar un equipo compacto, de bajo costo, manipulable y resistente para diagnosticar anemia de forma menos invasiva y rápida.

Por lo cual en este articulo mostramos el desarrollo y evaluación inicial de un equipo EIE (Figura 1) el cual pudo obtener lecturas en tejido sanguíneo y hallar diferencias en los espectros característicos de impedancia de cada persona voluntaria, a quienes previamente se les realizó un hemograma completo en la Clínica de la Universidad Nacional Mayor De San Marcos e inmediatamente se les extrajo una muestra sanguínea para el análisis respectivo en el equipo desarrollado. Con esto se espera contribuir en el desarrollo de tecnología médica para el diagnóstico de distintas enfermedades sanguíneas haciendo énfasis en la portabilidad de este equipo.

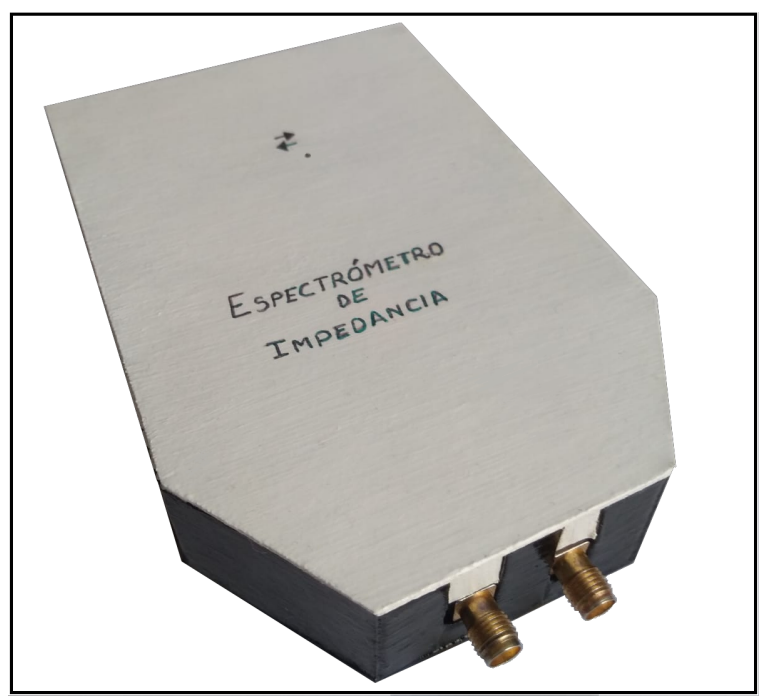

Figura 1: Equipo compacto EIE desarrollado durante la investigación

\section{Materiales y Métodos}

Inicialmente se desarrolló un diseño de un equipo compacto que concibiera la lectura de impedancia eléctrica utilizando un Analog Discovery 2 como base del circuito debido a su propiedad multifuncional, de bajo costo y fácil accesibilidad. Habiendo desarrollado el circuito interno del equipo, este se ensambló dentro de un case impreso en 3D el cual fue modelado en los programas Freecad [Fre20] y Tinkercad [Tin20] (Figura 2).

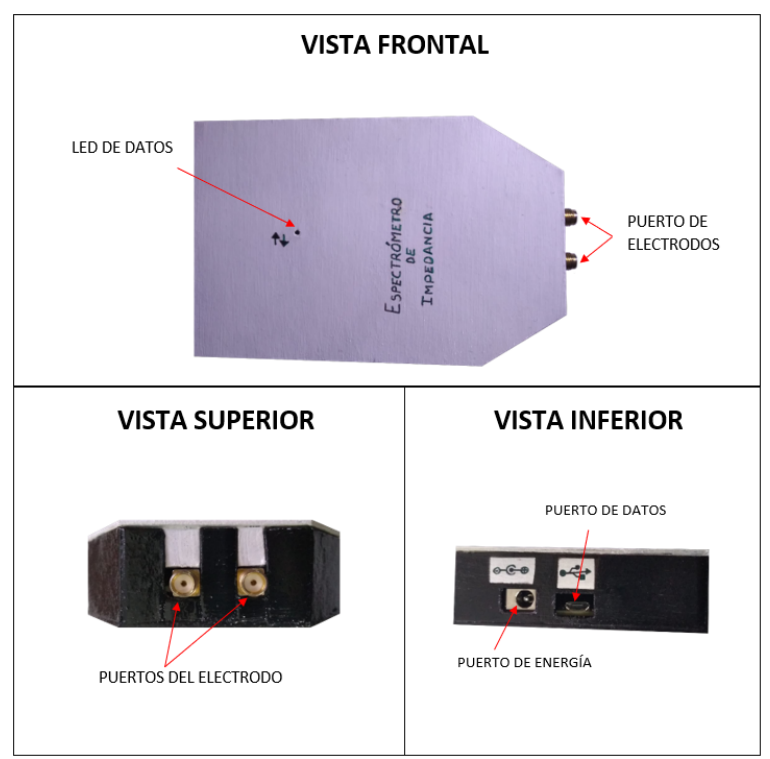

Figura 2: Vista frontal, superior e inferior del equipo EIE.

El circuito final del equipo EIE concibió dos terminales de salidas para la conexión del electrodo logrando un rango de frecuencia de $200 u \mathrm{~Hz}$ a $25 \mathrm{MHz}$ y con un rango de amplitud de diferencia potencial de $0 \mathrm{~V}$ a $5 \mathrm{~V}$, en la Figura 3 se muestra el diagrama de bloques correspondiente al circuito.

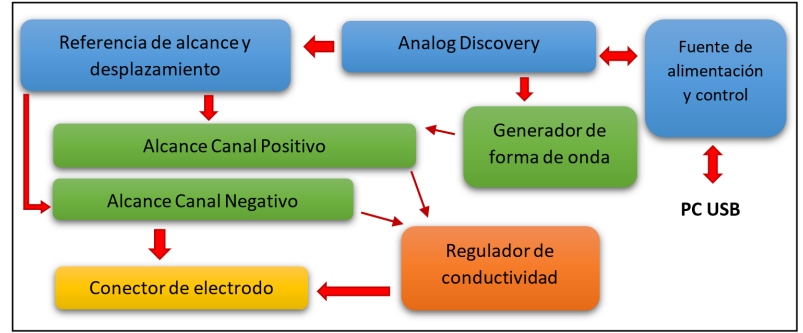

Figura 3: Diagrama de bloques del circuito interno del equipo EIE.

En paralelo, se diseñó también el conector (Figura 4) que fue adaptado para el uso de tiras reactivas de un glucómetro Accu-Chek@ como electrodos de lectura (Figura 5) con el fin de facilitar el uso de las tiras reactivas, ya que estas no pueden ser soldadas directamente debido 
a que son muy sensibles al calor y no pueden ser reutilizadas a causa de los parámetros de sanidad brindados por el Ministerio de Salud (MINSA) [Min13].

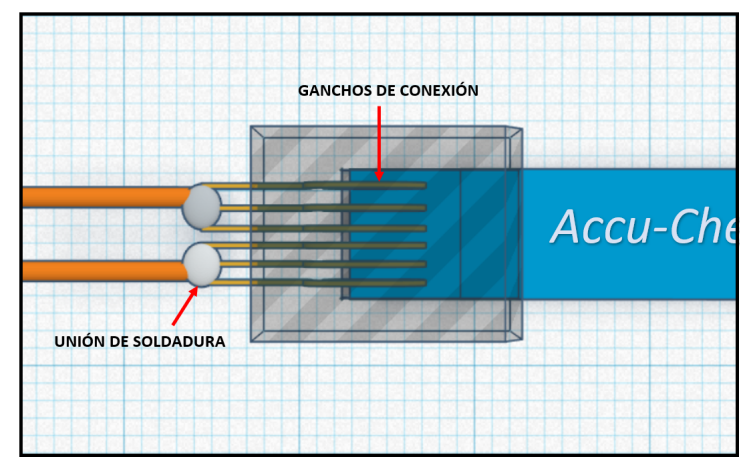

Figura 4: Diseño del adaptador para la tira reactiva.

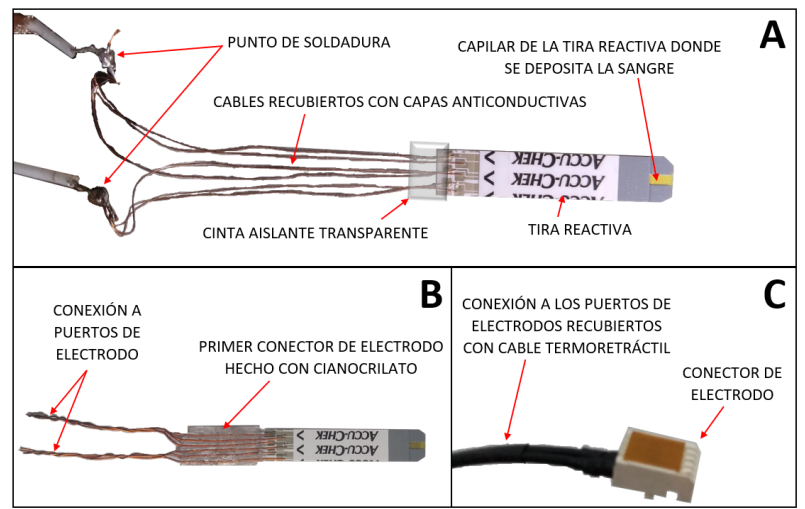

Figura 5: Foto de los conectores elaborados durante la experimentación. (A) Primer modelo de conector concebido utilizando cintas aislantes para sujetar los cables interdigitados. (B) Segundo modelo de conector utilizando una base de cianoacrilato para el ajuste de los interdigitados. (C) Modelo final del conector adaptador previamente modelado en 3D (Fig. 4).

Previamente, para verificar la funcionalidad, se elaboró un electrodo de calibración con un circuito R-C en paralelo que brindó un diagrama de Nyquist que concuerda con la literatura [Ora08],

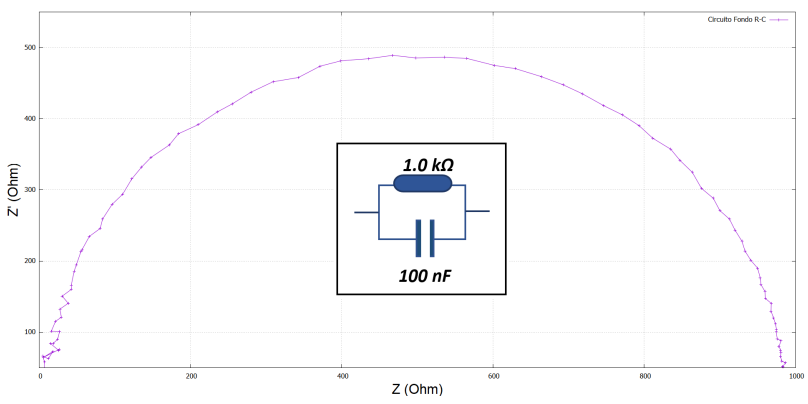

Figura 6: Representación gráfica con diagrama de Nyquist del circuito R-C en paralelo.

\begin{tabular}{lcr}
\hline Frecuencia $(\mathrm{Hz})$ & $\mathrm{Z}(\Omega)$ & $\mathrm{Z}^{\prime}(\Omega)$ \\
\hline 50.0 & 983.62 & 34.84 \\
$50.0 \times 10^{3}$ & 1.14 & 24.29 \\
\hline
\end{tabular}

Tabla 1: Los datos resistivos real e imaginario de impedancia obtenidos para un rango de frecuencias $(\mathrm{Hz})$, mostrado en la gráfica anterior (Figura 6).

Además de obtener como resultado los diagramas respectivos de Bode para la variación de la fase (Figura 7) y para el módulo de la impedancia (Figura 8), ambos respecto a la frecuencia.

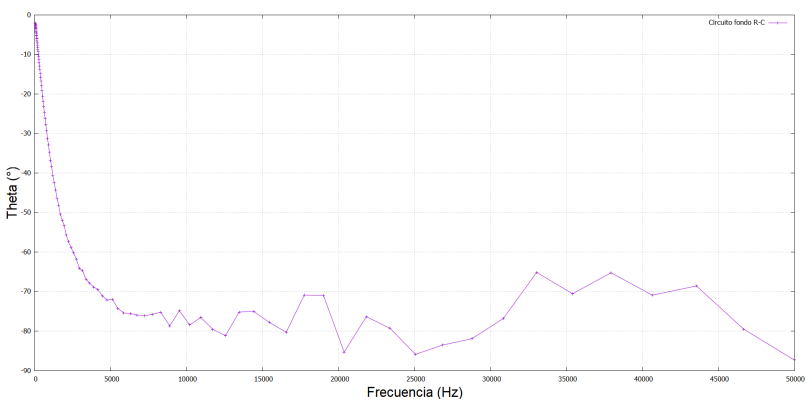

Figura 7: Representación gráfica de Fase vs Frecuencia.

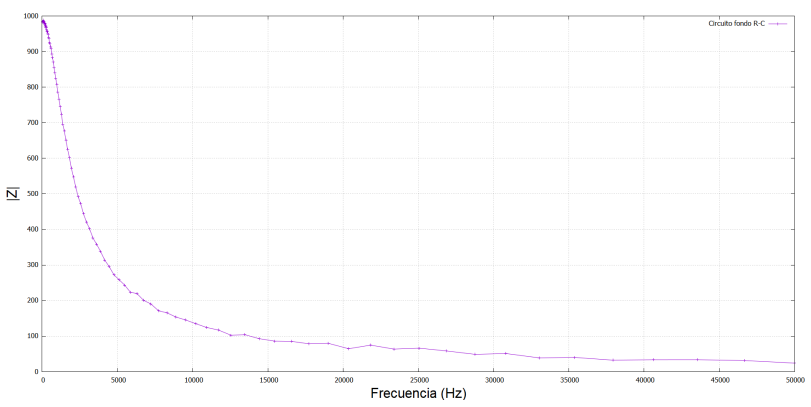

Figura 8: Representación gráfica de Amplitud de Impedancia vs Frecuencia.

\begin{tabular}{lcr}
\hline Frecuencia $(\mathrm{Hz})$ & $|\mathrm{Z}|$ & $\operatorname{Theta}\left({ }^{\circ}\right)$ \\
\hline 50.0 & 984.24 & -2.02 \\
$50.0 \times 10^{3}$ & 24.32 & -87.29 \\
\hline
\end{tabular}

Tabla 2: Datos del ángulo de fase $\left(\theta^{\circ}\right)$ y amplitud de impedancia (|Z|) aplicado a un rango de frecuencias (Hz), mostrado en la gráficas anteriores (Figuras 7,8).

Se observa claramente que el espectro obtenido para la fase presenta un notorio ruido debido a las frecuencias con las que se trabajó además del alto nivel de la resistencia perteneciente al circuito, sin embargo, para nuestro análisis esto no afecta de manera clara los resultados en 
el gráfico de Nyquist. Posteriormente, se evaluó los parámetros de extracción sanguínea por el método de punción capilar, el cual nos brinda con mayor precisión $0,6 \mu \mathrm{L}$ de sangre. Con esto, se inició el proceso experimental con 10 personas voluntarias de sexo masculino libres de VIH, Hepatitis, Brucelosis y enfermedad de Chagas. Se resalta que se escogió las muestras de este sexo con el fin de evitar parámetros de medida como los de menstruación y embarazo.

El equipo realizó las lecturas de EIE en un rango de frecuencias de $50 \mathrm{kHz}$ a $25 \mathrm{MHz}$, aplicando una diferencia de potencial regulada de $100 \mathrm{mV}$, encontrando así datos satisfactorios en la caracterización de la sangre y el correcto funcionamiento del equipo como parte inicial para un futuro equipo de diagnóstico.

\section{Resultados y Discusión}

El análisis del espectro característico propuesto para el estudio del comportamiento eléctrico del tejido sanguíneo está en relación a su respuesta en frecuencias de 50 $\mathrm{kHz}-25 \mathrm{MHz}$, al ser aplicada una diferencia de potencial constante bajo de $100 \mathrm{mV}$. Para ello se tiene en consideración que todos los electrodos presentan una capacitancia denominada "capacitancia de doble capa" $\left(C_{d l}\right)$. Así mismo, en una celda electroquímica existe una resistencia eléctrica asociada a la resistencia del electrolito denominada "Resistencia de la solución" $\left(R_{\text {sol }}\right)$, el cual produce una variación en la impedancia total del sistema [Gon12].

Se observa que la sangre se comporta como un circuito R-C en paralelo por la composición de la membrana celular, ya que la bicapa lipídica cumple la función de capacitor y los canales iónicos de conductores (Figura 9)

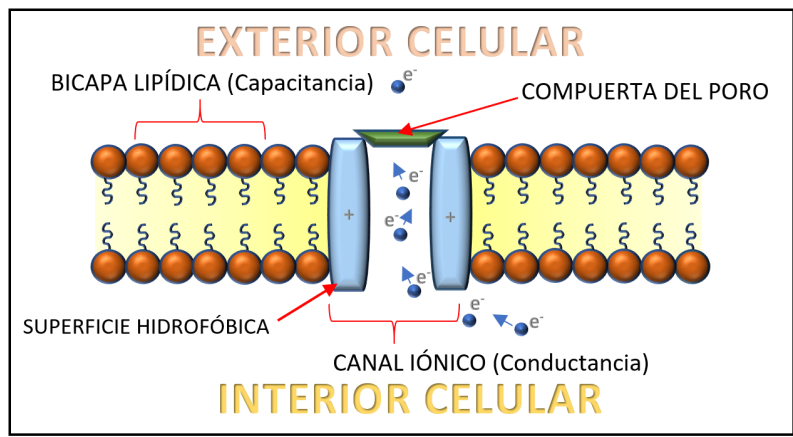

Figura 9: Comportamiento eléctrico de la membrana celular.

Debido a su comportamiento electrolítico, es necesario agregar al modelo eléctrico de la membrana celular el $R_{\text {sol }}$, obteniendo así el circuito presente en la Figura 10.

Para un modelo más realista en el comportamiento del tejido sanguíneo como circuito es necesario introducir un elemento de constante de fase (CPE), donde el CPE modela la impedancia de la doble capa debido al comportamiento no ideal de la capacitancia del electrodo:

$$
Z_{C P E}=\frac{1}{(j \omega)^{\phi} T}
$$

donde $\omega$ es la frecuencia angular aplicada, $j=$ $(-1)^{1 / 2}, T$ representa una relación con la capacitancia del electrodo y $\phi$ es un parámetro relacionado a la desviación de la recta capacitiva, en donde si este valor toma un máximo de 1, la impedancia del electrodo sería puramente capacitiva [Laz14].

Gracias a este concepto del comportamiento eléctrico del tejido sanguíneo, se puede realizar su representación algebraica Eq. (2), teniendo la impedancia total expresada como:

$$
Z=R_{\text {sol }}+\frac{R}{1+R(j \omega)^{\phi} T}
$$

donde $R_{\text {sol }}$ es la resistencia de la solución.

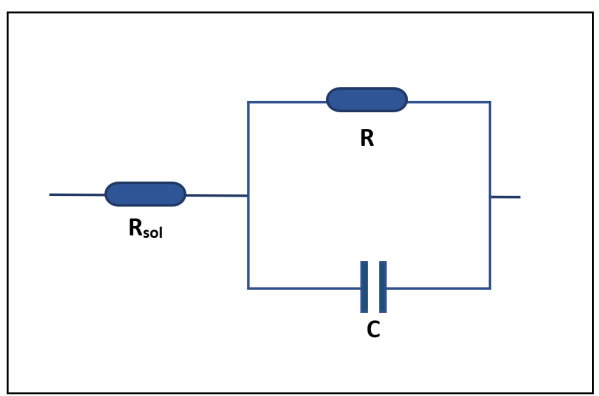

Figura 10: Circuito para soluciones electrolíticas.

Con las bases matemáticas y biofísicas ya establecidas, se esperaba obtener como resultado un diagrama de Nyquist similar al de la Figura 11, durante las lecturas en muestras sanguíneas [Vel12].

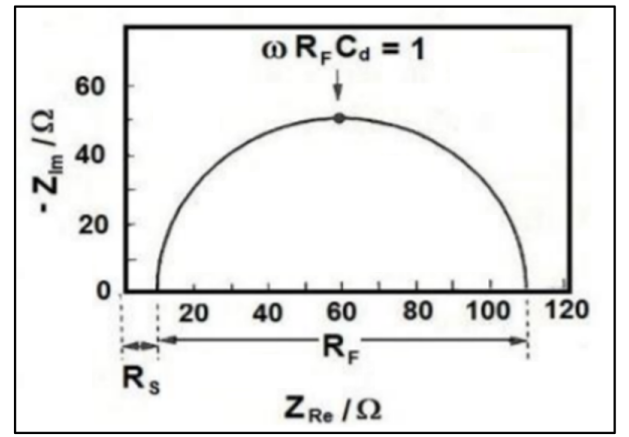

Figura 11: Representación en el plano complejo de la impedancia de una interface electroquímica [Vel12].

Para ello, a los pacientes voluntarios se les realizó un hemograma completo, donde se analizó la cantidad de $\mathrm{Hb}(\mathrm{gr} / \mathrm{dl})$ en sangre que tenían. En paralelo, utilizando parte de la misma muestra sanguínea se procedió a realizar una lectura de impedancia mediante el equipo EIE compacto. 


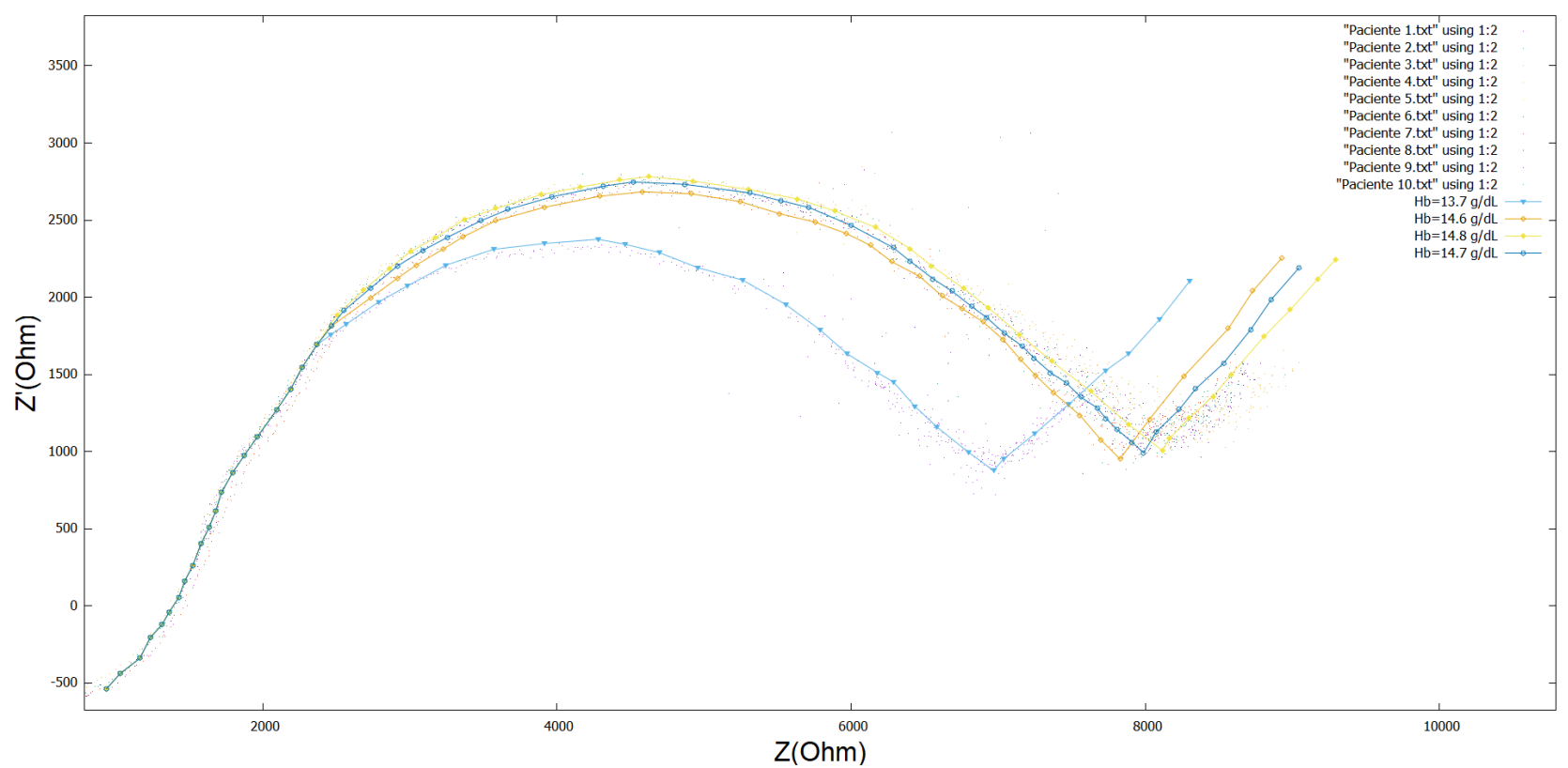

Figura 12: Espectro característico en sangre con su respectivo valor de hemoglobina en un rango de fracuencia de $50 \mathrm{kHz}$ a $25 \mathrm{MHz}$.

Los espectros característicos de los pacientes sanos tienen niveles normales de $\mathrm{Hb}$ en la sangre equivalentes a $13,7 \mathrm{~g} / \mathrm{dL}, 14,6 \mathrm{~g} / \mathrm{dL}, 14,7 \mathrm{~g} / \mathrm{dL}, 14,8 \mathrm{~g} / \mathrm{dL}$ (Figura 12).

Se observó que los espectros correspondientes al comportamiento eléctrico de la sangre corresponden con mayor semejanza al circuito Randles [Vel12] (Figura 13) y no al circuito que se planteó mostrado en las Figuras 10,11. Esto indica que hay una tendencia a responder a frecuencias más bajas (Figura 14).

\begin{tabular}{lcc}
\hline $\mathrm{Hb}(g / d L)$ & $\mathrm{Z}(\Omega)$ & $\mathrm{Z}^{\prime}(\Omega)$ \\
\hline 13.7 & 8301.00 & 2105.43 \\
14.6 & 8924.59 & 2252.11 \\
14.7 & 9045.54 & 2192.93 \\
14.8 & 9290.55 & 2242.49 \\
\hline
\end{tabular}

Tabla 3: Los datos resistivos real e imaginario de impedancia relacionado con su respectivo dato de cantidad de $\mathrm{Hb}$ en sangre a una frecuencia de $50.0 \mathrm{kHz}$, mostrado en la gráfica anterior (Figura 12).

De la gráfica de Nyquist anterior (Figura 12) se puede observar un punto de inflexión pronunciado que a simple vista marca una diferencia característica entre cada uno de los distintos espectros para cada $\mathrm{Hb}$ en sangre el cual podría tratarse de la impedancia de Warburg, llevándonos a pensar en el circuito de Randles. Cabe mencionar que este evento tuvo lugar en un rango de frecuencia de
$140 \mathrm{kHz}$ a $170 \mathrm{kHz}$ y la distancia entre cada punto es mas visible en el eje $\mathrm{Z}(\mathrm{Ohm})$.

\begin{tabular}{lcr}
\hline $\mathrm{Hb}(g / d L)$ & $\mathrm{Z}(\Omega)$ & \multicolumn{1}{c}{$\mathrm{Z}^{\prime}(\Omega)$} \\
\hline 13.7 & 6967.88 & 878.51 \\
14.6 & 7828.19 & 954.25 \\
14.7 & 7988.04 & 994.64 \\
14.8 & 8115.55 & 1011.48 \\
\hline
\end{tabular}

Tabla 4: Los datos resistivos real e imaginario de impedancia obtenidos para un rango de frecuencias de $140 \mathrm{kHz}$ a 170 $\mathrm{kHz}$, mostrado en la gráfica anterior (Figura 12).

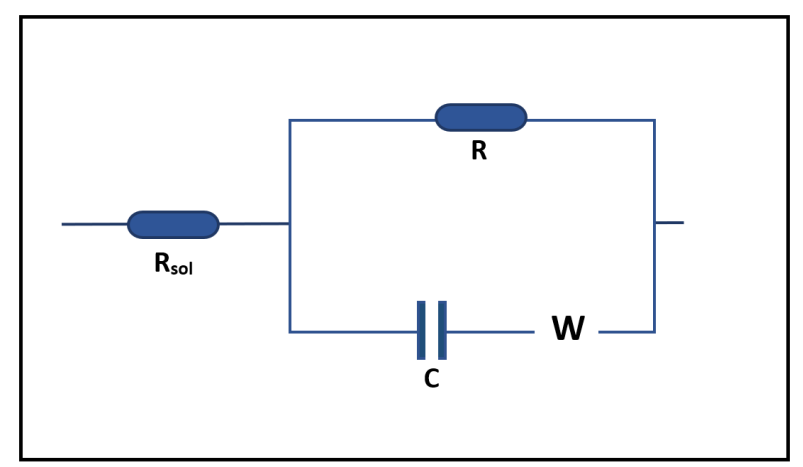

Figura 13: Circuito Eléctrico Randles donde W representa la impedancia de Warburg. 


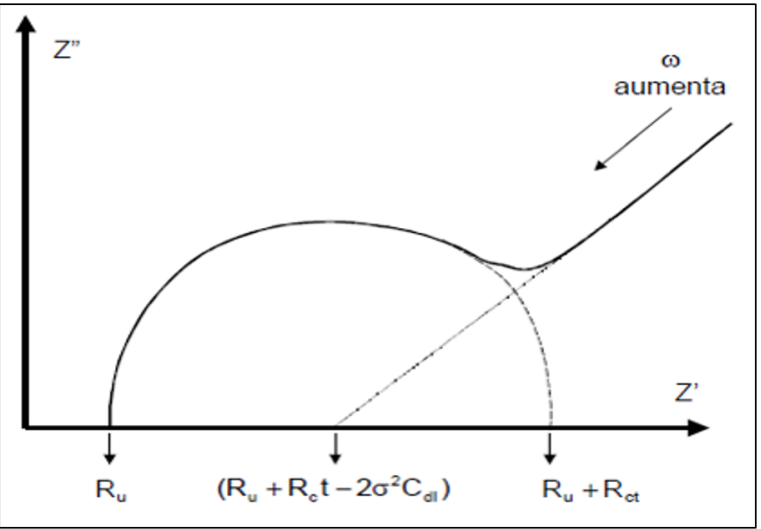

Figura 14: Representación plana compleja de la impedancia de una interfaz electroquímica utilizando impedancia Warburg (W) [Men10].

\section{Conclusiones}

El análisis de los datos indica que los niveles de $\mathrm{Hb}$ influyen en el espectro de impedancia debido a las diferencias observadas entre los diagramas de Nyquist durante la etapa experimental. (Figura 12)

También podemos concluir el cambio en el modelado del circuito sanguíneo debido a la línea de tendencia presentada en los resultados experimentales, además del cambio en su representación algebraica, donde (Eq.3,4) representa el comportamiento de los espectros de impedancia cuando al alcanzar altas frecuencias la impedancia de Warburg tiene un pequeño valor en comparación con $\mathrm{R}$ [Gon12].

$$
\begin{aligned}
Z & =R_{\text {sol }}+\frac{R}{1+\omega^{2} C_{d l}^{2} R^{2}} \\
Z^{\prime} & =R_{\text {sol }}+\frac{C_{d l} R^{2} \omega}{1+\omega^{2} C_{d l}^{2} R^{2}}
\end{aligned}
$$

Además, en el tratamiento de frecuencias bajas, cuando tiende a cero, los valores de la impedancia total se describen mediante las siguientes ecuaciones:

$$
\begin{aligned}
& Z=R_{\text {sol }}+R+\sigma \omega^{-1 / 2} \\
& Z^{\prime}=\sigma \omega^{-1 / 2}+2 \sigma^{2} \omega_{d l}
\end{aligned}
$$

Se obtiene de la combinación de estas dos últimas ecuaciones lo siguiente:

$$
Z^{\prime}=Z-R_{\text {sol }}-R+2 \sigma^{2} C_{d l}
$$

Esta ecuación (Eq.7) al ser graficada en el plano complejo se obtiene una recta con pendiente unitaria con el cual se puede determinar el coeficiente de transferencia de masa $(\sigma)$.

Tomando la premisa del circuito de Randles, la línea de tendencia en las respuestas de baja frecuencia podría confirmarse que proporcionó satisfactoriamente la caracterización esperada durante la comparación en las muestras obtenidas de 10 pacientes con 13,6 g/dL, 13,7 $g / d L$ y $13,8 g / d L$, además se pudo realizar una muestra simulada correspondiente a un paciente de anemia con $12,7 \mathrm{~g} / \mathrm{dL}$ de hemoglobina en sangre a partir de los datos reales anteriormente obtenidos, este último evaluado con el fin de desarrollar a futuro un nuevo método de diagnóstico. (Figura 14).

Con esto, el desarrollo de un equipo de diagnóstico que puede ser capaz de diferenciar las lecturas entre una persona sana, propensa a tener anemia y anémica, se hace posible en el futuro. Para ello, también se ve necesario diseñar una interfaz digital, que pueda automatizar la extracción de datos obtenidos por el equipo EIE; esta interfaz digital se viene desarrollando en Python debido a su particularidad de ser multiplataforma. También se concibe adaptar un potenciostato para ayudar al equipo a obtener mediciones a frecuencias muy inferiores a las disponibles actualmente, además de incluir un tercer terminal a la conexión de electrodos para lograr una mejor resolución en sus mediciones.

Debemos mencionar que este equipo con los datos que proporcionó podría ser utilizado también para la detección de otras enfermedades como la leucemia [Ber05a], Salmonella Typhimurium, Malaria [Fab09], incluso virus como el H5N1 [Ber09]; este ultimo inspiró para la propuesta teórica-experimental titulada "Low cost and fast diagnostic equipment for COVID-19 using electrical impedance spectroscopy" ganando el segundo galardón en Global First Penguin Club Conference (G1PEC), el cual busca el desarrollo mas rápido y de bajo costo para detectar COVID-19 mediante espectroscopia de impedancia eléctrica.

\section{Agradecimientos}

Agradecemos al Grupo de Investigación del I.E. Gabriela Mistral UGEL 03 y a la Mg. Yuly Oyanguren B. por proporcionarnos sus instalaciones para la aplicación experimental de los conocimientos previos. Así también agradecemos la colaboración de la Bach. Sara V. Diaz Arrue por su análisis para las enfermedades que implican la disminución de $\mathrm{Hb}$ en sangre como la anemia y a la estudiante de la Facultad de Genética y Biotecnología Nicole S. Morante Calderón por sus recomendaciones para el análisis de elaborar equipos de diagnóstico. 


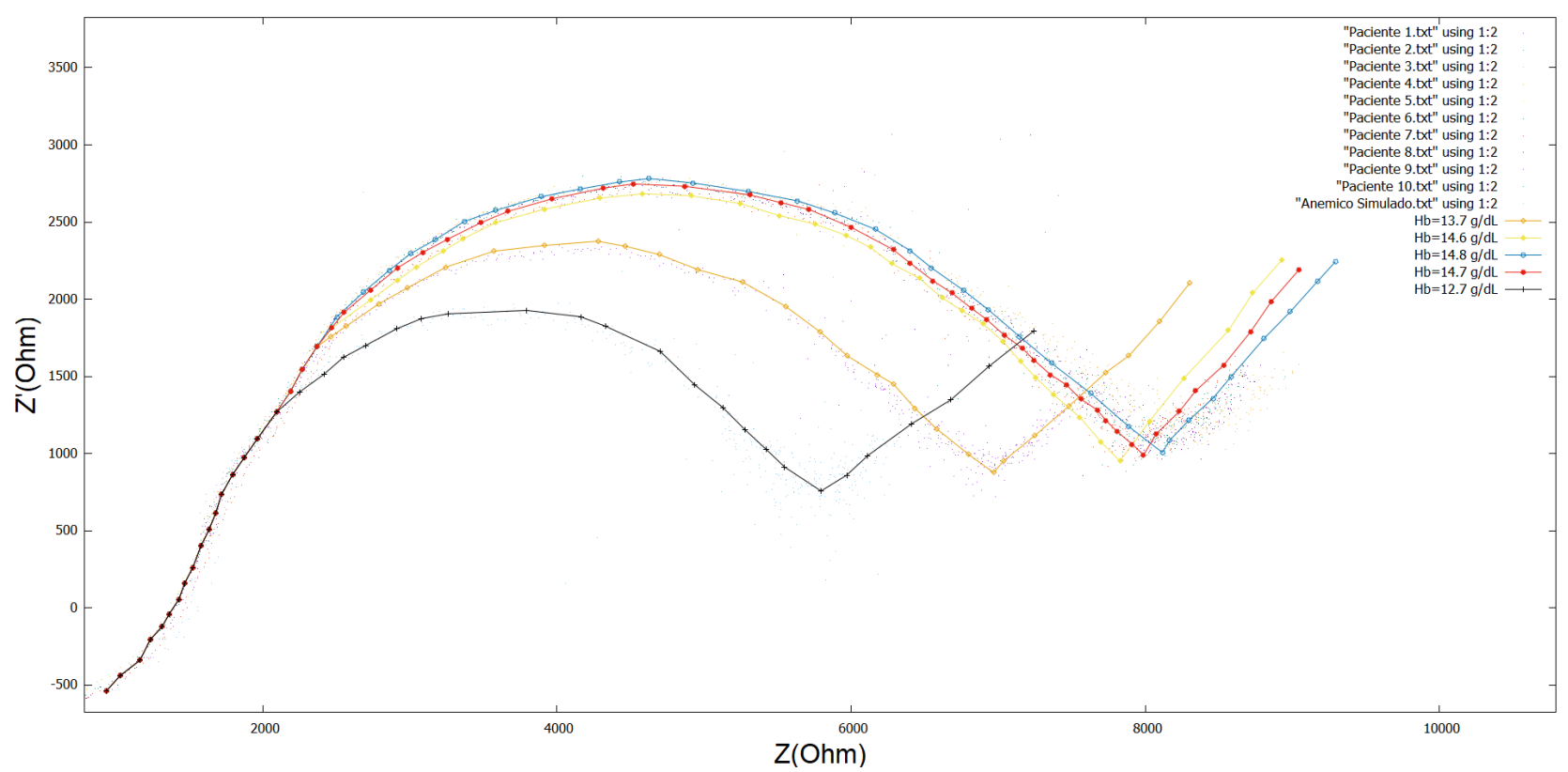

Figura 15: Espectro de impedancia sanguínea característico para 10 pacientes con diferencias de Hb en la sangre y uno simulado con $\mathrm{Hb}=12.7 \mathrm{~g} / \mathrm{dL}$ que tienen el punto de corte en el rango de frecuencia de $140 \mathrm{kHz}-170 \mathrm{kHz}$.

\section{Referencias}

[Alm12] Almagiá, A., Lizana, P., Rodriguez, R., Gallardo, L., Nieto, C., Verdejo, S., Ivanovic, D., Binvignat,O. (2012). Predicción de Porcentaje de Masa Adiposa a través de Impedancia Bio-Eléctrica y Método Antropométrico. International Journal of Morphology, 30(3), 872-876.

[And05] Anders, H. (2005). Influence of electrolyte in transport and recombination in dye-sensitized solar cells studied by impedance spectroscopy. Solar Energy Materials and Solar Cells, 87, 117-131.

[And10] Andriolo, A., Alvaro, R., Carlos, F., Ballarati, I., Maria M. (2010). Recomendaciones de la Sociedad Brasileña de Patologia Clínica Medicina Laboratorial para la extracción de sangre venosa. Brasil.

[Bal19] Baltuano, O. (2019). Desarrollo y evaluación de un analizador compacto de impedancias para aplicaciones médicas y biológicas (Tesis de Maestría). Universidad Nacional de Ingenieria, Lima, Perú.

[Ber05a] Bernal, J., Guerrero, C., y Hernández, F. (2005 a). Determinación de las propiedades eléctricas en tejido sanguíneo. Universidad Autónoma de Nuevo León, 510-515.

[Ber05b] Bernal, J. M. Sosa, J., Bernaln, M. Jiménez, C., Hernández, G., Gutiérrez, M., Huerta, J., Vi- llagómez C., and P. Palomares (2005 b). Magnetic Field Influence on Electrical Properties of Human Blood Measured by Impedance Spectroscopy. Bioelectromagnetics, 564-570.

[Ber09] Berghman, L., Ronghui, Y., Lassiter, Y., Billy, S., L., Walter, B. (2009). Interdigitated array microelectrode based impedance immunosensor for detection of avian influenza virus H5N1. Talanta, 159-164.

[Cal80] Callow, L., Dawson, J., and Hladky, K. (1980). Corrosion Rates from Impedance Measurements: An Introduction. British Corrosion Journal 15, 20-25, United Kingdom.

[Cau85] Caughey, W., Kawanishi, S., and Watkins, J. (1985). Autoxidation reactions of hemoglobin a free from other red cell components: A minimal mechanism. Biochemical and biophysical research communications, 742-748.

[Fab09] Fabre, P., Launay, J., Nepveu, F., Ribaut, C., Reybier, K., Reynes, O., and Valentin, A. (2009). Electrochemical impedance spectroscopy to study physiological changes affecting the red blood cell after invasion by malaria parasites. Biosensors and Bioelectronics, 2721-2725.

[Fre20] FreeCad (No. de versión 0.18.4). (2020). Linux. DigitalOcean. 
[Got96] Gottesfeld, S. (1996). Characterization of Polymer Electrolyte Fuel Cells Using AC Impedance Spectroscopy. Journal of The Electrochemical Society, 143, 587-599.

[Gom07] Gómez, J., Múnez, C., Poza, P., Salazar, A., y Utrilla, V. (2007). Análisis de la degradación de recubrimientos de barrera térmica por espectroscopía de impedancia electroquímica. Boletin de la sociedad española de cerámica y vidrio, 46, 232-239.

[Gon12] Gonzáles, J. (2012). 1.1 Espectroscopía de impedancia eléctroquímica (EIE). Universidad Autónoma de Madrid, España.

[Gri04] Griffis, C., Johnson, M., Li, Y., and Yang, L. (2004). Interdigitated microelectrode (IME) impedance sensor for the detection of viable Salmonella typhimurium. Biosensors and Bioelectronics, 1139-1147.

[Ine19] INEI. (2019). Indicadores de Resultados de los Programas Presupuestales, Primer Semestre 2019. Encuesta Demográfica y de Salud Familiar (Resultados Preliminares al $50 \%$ de la muestra). Instituto Nacional de Estadística e Informática, Lima.
[Laz14] Lazia, A. (2014). Electrochemical Impedance Spectroscopy and its Applications. Canada: Springer.

[Mas10] Masot, R. (2010). Desarrollo de un sistema de medida basado en espectroscopía de impedancia para la determinación de parámetros fisicoquímicos en alimentos. Valencia, España.

[Men10] Mendoza, J., Durán, R., y Genescá, J. (2010). Espectroscopía de Impedancia Electroquímica en Corrosión. México.

[Min13] (MINSA), Midori de Habich (2013). Procedimientos de Laboratorio. Lima, Perú.

[Ora08] Orazem, M., and Tribollet, B. (2008). Electrochemical Impedance Spectroscopy . New Jersey: WILEY.

[Tin20] Tinkercad. (2020). Linux. Autodesk.

[Vel12] Velasco, J. G. (2012). Espectroscopía de impedancia electroquímica (EIE). España. 